\title{
Transient analysis on track circuits in crack state
}

\author{
Zhao Bin \\ School of Automation \& Electrical Engineering, Lanzhou Jiao tong University, Lanzhou, China. 730070
}

\begin{abstract}
The transient transformation of track circuit between the normal state and crack state is analyzed. Firstly, the model of crack state of track circuit in the complex frequency domain is established. According to the boundary conditions of crack state of track circuit, the expression of current traveling wave in the receiving end is obtained in complex frequency domain. Based on it, the numerical solution in the time domain is got by FFT\&IFFT conjunction with quotient-difference (Q-D) algorithm. Then, the steady state of circuit in normal state is taken as state of zero moment of track circuit, the transient process from normal state transform into crack state is solved. Thirdly, the Electro-Magnetic Transient Program (EMTP) model of crack operation is established to prove that the transient analysis result is correct. It provides a power theoretical basis to distinguish the operation state of track circuit by transient analysis.
\end{abstract}

\section{Introduction}

In order to analyze transient process of track circuits in crack state, there are three problems will be solved. The first is how to solve the transmission line equation, the second is how to get boundary conditions according to rail fracture state equivalent model, the three is that crack state complete response analysis with the initial condition of steady values in normal state.

The solution of distortion transmission lines was solved by analytical method in [4]. However, this method is not suitable to track circuit, as the track circuit belongs to distortion transmission lines. The solution of track circuit transmission lines equation is difficultly transformed by inverse Fourier transform (IFFT) or inverse Laplace transform(ILT), so it is difficult to solve track circuit transmission line equation by analytical method. The track circuit is analyzed under specific loads in time domain in [5], but the analytical solutions has no general. The transmission line equation is solved by numerical method in most literatures. Numerical analysis have some weaknesses. For example, the order number of transfer function is higher, the computation complexity is bigger, and the problem is simplified relatively complicated[6-9]. FFT\&IFFT conjunction with quotient-difference(Q-D) algorithm does not refer the zeros and poles of transfer function, and the computation complexity is lower [10]. When the steady state of track circuit in normal state is taken as state of zero moment of track circuit in crack state, the transmission line is four order linearity non homogeneous partial differential equation. Based on the solution of high order linearity non homogeneous partial differential equation, fourth order indefinite integral which is difficult to solve will appear $[11,12]$.

\section{Analysis Of Crack States Of Track Circuit}

\subsection{The crack states model of track circuit}

The equivalent circuit model of track circuit crack state in complex frequency domain is shown in Fig.1.

Where: $u_{s}(s)$ is the sending end voltage source of track circuit, $Z_{1}(s)$ is internal impedance of track Where: $u_{s}(s)$ is the sending end voltage source of track circuit, $Z_{1}(s)$ is internal impedance of track circuit, $Z_{L}(s)$ is the receiving impedance, zero coorinates is crack point, $Z_{\mathrm{b}}(s)$ is equivalent impedance of the crack point.

*Corresponding author: zhaobin@ mail.lzjtu.cn 


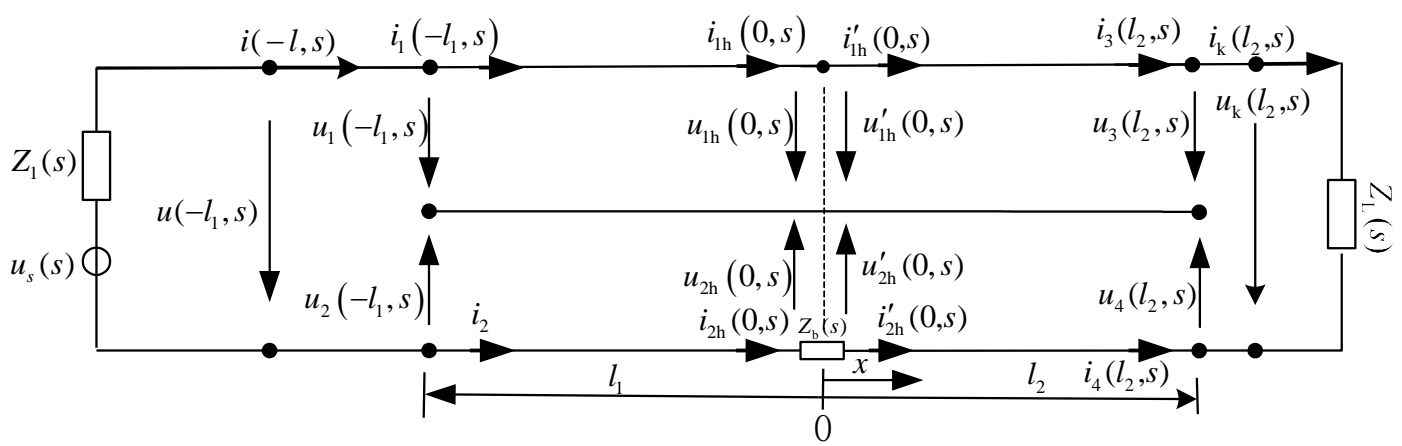

Figure.1. The equivalent circuit model of track circuit crack states in complex frequency domain

\subsection{The crack states expression of track circuit travelling wave}

According to reference [5], in the initial condition of zero, the expression of voltage and current of each rail to ground can be written to (1)-(4):

$$
\begin{aligned}
& u_{1}(x, s)=F_{1}(s) \mathrm{e}^{\gamma_{1}(s)}+F_{2}(s) \mathrm{e}^{-\gamma_{1}(s)} \\
& +F_{3}(s) \mathrm{e}^{\gamma_{3}(s)}+F_{4}(s) \mathrm{e}^{-\gamma_{3}(s)} \\
& u_{2}(x, s)=F_{1}(s) \mathrm{e}^{\gamma_{1}(s)}+F_{2}(s) \mathrm{e}^{-\gamma_{1}(s)} \\
& -F_{3}(s) \mathrm{e}^{\gamma_{3}(s)}-F_{4}(s) \mathrm{e}^{-\gamma_{3}(s)} \\
& i_{1}(x, s)=\frac{-1}{Z B_{1}(s)} F_{1}(s) \mathrm{e}^{\gamma_{1}(s) x}+\frac{1}{Z B_{1}(s)} F_{2}(s) \mathrm{e}^{-\gamma_{1}(s) x} \\
& -\frac{1}{Z B_{2}(s)} F_{3}(s) \mathrm{e}^{\gamma_{3}(s) x}+\frac{1}{Z B_{2}(s)} F_{4}(s) \mathrm{e}^{-\gamma_{3}(s) x} \\
& i_{2}(x, s)=\frac{-1}{Z B_{1}(s)} F_{1}(s) \mathrm{e}^{\gamma_{1}(s) x}+\frac{1}{Z B_{1}(s)} F_{2}(s) \mathrm{e}^{-\gamma_{1}(s) x} \\
& +\frac{1}{Z B_{2}(s)} F_{3}(s) \mathrm{e}^{\gamma_{3}(s) x}-\frac{1}{Z B_{2}(s)} F_{4}(s) \mathrm{e}^{-\gamma_{3}(s) x}
\end{aligned}
$$

Where: $F_{1}(s) \sim F_{4}(s)$ are integral coefficient of track circuit in normal state, $Z B_{2}(s)$ is the characteristic impedance between two of rail, $Z B_{1}(s)$ is the characteristic impedance from arbitrary rail to ground. $\gamma_{1}(s)$ is propagation constant of rail to ground, $\gamma_{3}(s)$ is propagation constant of between two rail. The integral coefficient of the section $l_{1}$ can be described by $K_{1}(s) \sim K_{4}(s)$. The integral coefficient of the section $l_{2}$ can be described by $H_{1}(s) \sim H_{4}(s)$.

$K_{1}(s) \sim K_{4}(s)$ and $H_{1}(s) \sim H_{4}(s)$ are obtained by using the boundary conditions of track circuit in crack state. According to the condition1 condition5,

(5) (12) can be got.

Condition 1 : when $x=0$,

$$
i_{2 \mathrm{~h}}(0, s)=i_{2 \mathrm{~h}}{ }^{\prime}(0, s)=\frac{u_{2 \mathrm{~h}}(0, s)-u_{2 \mathrm{~h}}{ }^{\prime}(0, s)}{Z_{\mathrm{b}}(s)} .
$$

Condition2: when $x=0$,

Condition3: when $x=0$,

$$
u_{\mathrm{lh}}(0, s)=u_{\mathrm{lh}}^{\prime}(0, s) \text {. }
$$

Condition4: when $x=-l_{1}$

$$
i_{\mathrm{lh}}(0, s)=i_{\mathrm{lh}}^{\prime}(0, s) .
$$

$$
\begin{gathered}
\frac{i_{1}\left(-l_{1}, s\right)-i_{2}\left(-l_{1}, s\right)}{2} Z_{1}(s)=u_{s}-\left[u_{1}\left(-l_{1}, s\right)-u_{2}\left(-l_{1}, s\right)\right] \\
i_{1}\left(-l_{1}, s\right)=-i_{2}\left(-l_{1}, s\right) .
\end{gathered}
$$

Condition5: when $x=l_{2}$

$$
\begin{gathered}
\frac{i_{3}\left(l_{1}, s\right)-i_{4}\left(l_{1}, s\right)}{2} \mathrm{Z}_{\mathrm{L}}(s)=u_{3}\left(l_{2}, s\right)-u_{4}\left(l_{2}, s\right) \\
i_{3}\left(l_{2}, s\right)=-i_{4}\left(l_{2}, s\right) .
\end{gathered}
$$

$$
\begin{gathered}
-K_{1}(s)+K_{2}(s)+\frac{Z B_{1}(s)}{Z B_{2}(s)} K_{3}(s)-\frac{Z B_{1}(s)}{Z B_{2}(s)} K_{4}(s) \\
=\frac{Z B_{1}(s)}{Z_{\mathrm{b}}(s)}\left[K_{1}(s)+K_{2}(s)-K_{3}(s)-K_{4}(s)-H_{1}(s)-H_{2}(s)+H_{3}(s)+H_{4}(s)\right] \\
-H_{1}(s)+H_{2}(s)+\frac{Z B_{1}(s)}{Z B_{2}(s)} H_{3}(s)-\frac{Z B_{1}(s)}{Z B_{2}(s)} H_{4}(s) \\
=\frac{Z B_{1}(s)}{Z_{\mathrm{b}}(s)}\left[K_{1}(s)+K_{2}(s)-K_{3}(s)-K_{4}(s)-H_{1}(s)-H_{2}(s)+H_{3}(s)+H_{4}(s)\right] \\
H_{1}(s)+H_{2}(s)+H_{3}(s)+H_{4}(s)=K_{1}(s)+K_{2}(s)+K_{3}(s)+K_{4}(s) \\
-H_{1}(s)+H_{2}(s)-\frac{Z B_{1}(s)}{Z B_{2}(s)} H_{3}(s)+\frac{Z B_{1}(s)}{Z B_{2}(s)} H_{4}(s) \\
=-K_{1}(s)+K_{2}(s)-\frac{Z B_{1}(s)}{Z B_{2}(s)} K_{3}(s)+\frac{Z B_{1}(s)}{Z B_{2}(s)} K_{4}(s)
\end{gathered}
$$




$$
\begin{gathered}
K_{4}(s)=k(s) u_{s} \mathrm{e}^{-\gamma_{3}(s) l_{1}}+n_{1}(s) K_{3}(s) \mathrm{e}^{-2 \gamma_{3}(s) l_{1}} \\
K_{2}(s)=K_{1}(s) \mathrm{e}^{-2 \gamma_{1}(s) l_{1}} \\
H_{2}(s)=H_{1}(s) \mathrm{e}^{2 \gamma_{1}(s) l_{2}} \\
H_{3}(s)=n_{2}(s) H_{4}(s) \mathrm{e}^{-2 \gamma_{3}(s) l_{2}}
\end{gathered}
$$

$n_{1}(s)$ is defined as $n_{1}(s)=\frac{Z_{1}(s)-2 Z B_{2}(s)}{2 Z B_{2}(s)+Z_{1}(s)}$, $n_{2}(s)$ is defined as $n_{2}(s)=\frac{Z_{L}(s)-2 Z B_{2}(s)}{2 Z B_{2}(s)+Z_{L}(s)}, k(s)$ is defined as $k(s)=\frac{Z B_{2}(s)}{2 Z B_{2}(s)+Z_{1}(s)}$.

(13),(14) can be obtained according to (5) (12)

$$
H_{3}(s)=\frac{\eta(s)-\alpha(s) b_{\text {bro }}(s)}{\mu(s)} n_{2}(s) \mathrm{e}^{-2 \gamma_{3}(s) l_{2}} u_{s}(s)(13)
$$

$$
H_{4}(s)=\frac{\eta(s)-\alpha(s) b_{\text {bro }}(s)}{\mu(s)} u_{s}(s)
$$

where:

$$
\begin{aligned}
& \alpha(s)=\frac{\mathrm{e}^{-2 \gamma_{1}(s) l_{1}}-1}{\mathrm{e}^{2 \gamma_{1}(s) l_{2}}-1} \quad \beta(\mathrm{s})=\frac{n_{2}(s) \mathrm{e}^{-2 \gamma_{3}(s) l_{2}}-1}{1-n_{1}(s) \mathrm{e}^{-2 \gamma_{3}(s) l_{1}}} \\
& \mu(s)=\frac{\left[m_{2}(s) \beta(s)-m_{3}(s) n_{1}(s) \beta(s) \mathrm{e}^{-2 \gamma_{3}(s) l_{1}}-m_{4}(s) n_{2}(s) \mathrm{e}^{-2 \gamma_{\mathrm{f}}(s) l_{2}}-m_{4}(s)\right]}{\left[\alpha(s) m_{1}(s) \mathrm{e}^{-2 \gamma_{\mathrm{f}}(s) l_{1}}-\alpha(s)+m_{4}(s)+m_{4}(s) \mathrm{e}^{2 \gamma_{f}(s) l_{2}}\right]} \\
& m_{1}(s)=\frac{\left[1-\alpha_{\mathrm{f}}(s)\right]}{\left[1+\alpha_{\mathrm{f}}(s)\right]}, m_{2}(s)=\frac{\left[k_{\mathrm{a}}(s)+\alpha_{\mathrm{f}}(s)\right]}{\left[1+\alpha_{\mathrm{f}}(s)\right]}, m_{3}(s)=\frac{\left[k_{\mathrm{a}}(s)-\alpha_{\mathrm{f}}(s)\right]}{\left[1+\alpha_{\mathrm{f}}(s)\right]}, m_{4}(s)=\frac{\alpha_{\mathrm{f}}(s)}{\left[1+\alpha_{\mathrm{f}}(s)\right]} \\
& k_{\mathrm{a}}(s)=\frac{Z B_{1}(s)}{Z B_{2}(s)}, \quad \alpha_{\mathrm{f}}(s)=\frac{Z B_{1}(s)}{Z_{\mathrm{b}}(s)} .
\end{aligned}
$$

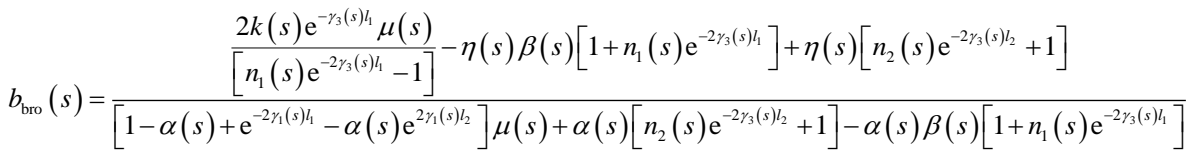

When $0<x \leq l_{2}$, in arbitrary point of track circuit, the expression of voltage $u(x, s)$ and current $i(x, s)$ are obtained in complex frequency domain: $u(x, s)=2 H_{3}(s) \mathrm{e}^{\gamma_{3}(s) x}-2 H_{4}(s) \mathrm{e}^{-\gamma_{3}(s) x}$

$i(x, s)=\frac{1}{Z B_{2}(s)}\left[H_{4}(s) \mathrm{e}^{-\gamma_{3}(s) x}-H_{3}(s) \mathrm{e}^{\gamma_{3}(s) x}\right]$

\subsection{The simulation of track circuit in crack state}

$Z_{\mathrm{b}}(s)$ is defined as the equivalent impedance of the crack point in crack model of track circuit. When $Z_{\mathrm{b}}(s) \rightarrow 0$, the track circuit operates in normal state, when $Z_{\mathrm{b}}(s)=\infty$, the track circuit operates in broken state, when $Z_{\mathrm{b}}(s) \in(0, \infty)$, the track circuit operates in crack state.

Transmission line whole response is the sum of zero state response with zero input response, zero input response can be calculated according to the principle that energy storage component of charging

and discharging is reciprocal. Zero input response is discharge process, which the steady-state value of the previous working is regard as the initial values of current work. So, the zero input response can be regarded that zero state response minus the steady state of track circuit $[13,14]$.

We assume the rail crack when track circuit energized after $10 \mathrm{~ms}, Z_{\mathrm{b}}(s)=Z \mathrm{~B}_{1}(s)$. The whole response current travelling wave in crack state is shown as Figure.2.

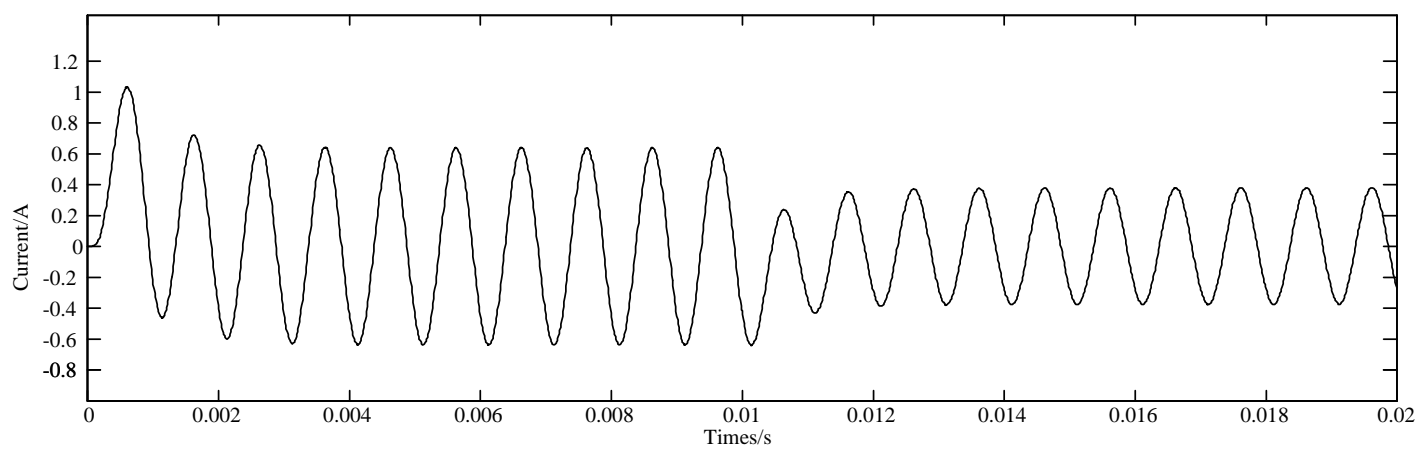

Figure2.The whole response current travelling wave in crack state

Form the figure2, in moment of the rail crack, it is a transient process that steady state of track circuit in normal state transmit to crack state.
In order to validate the result of theoretical analysis, the EMTP model is set as figure 3 and the simulation result is shown as figure4 [15]. 


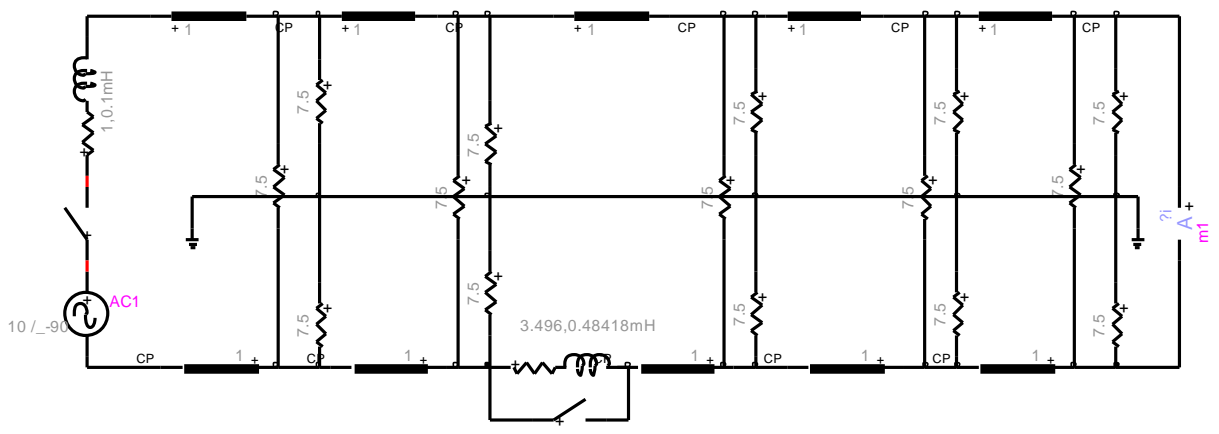

Figure.3. EMTP model of track circuit in crack state

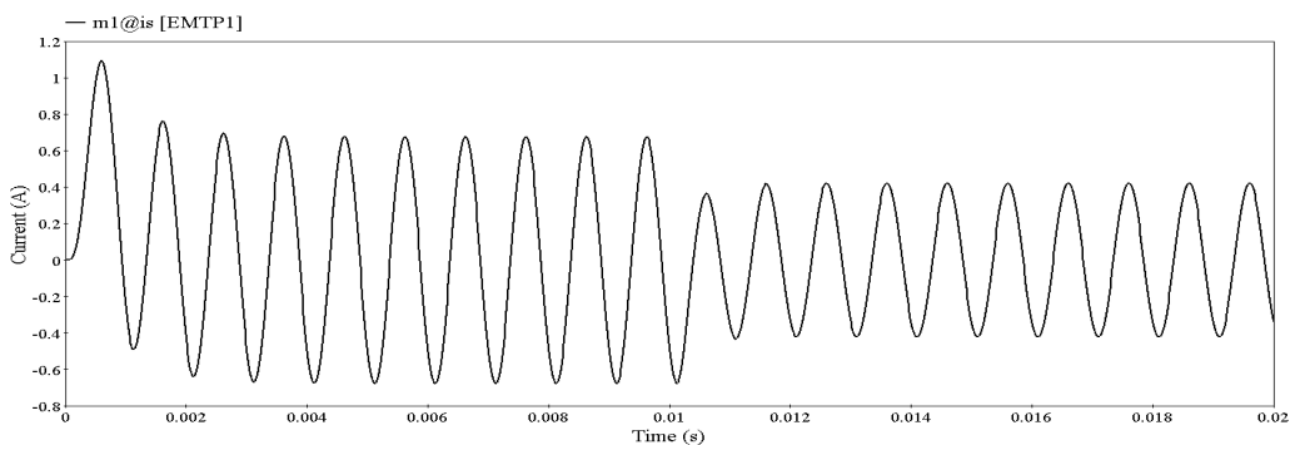

Figure.4. The whole response current travelling wave in crack state based on EMTP model

Comparing with Fig. 2 and Fig.4, the transient analysis method of track circuit in crack operation is correct.

\section{Conclusion}

The equivalent circuit model of track circuit crack states in complex frequency domain was established. Based on it, the expression of current (voltage) travelling wave of track circuit in crack operation was obtained in complex frequency, The numerical solution was solved by FFT\&IFFT conjunction with quotient-difference (Q-D) algorithm. Simulation result show that the method related in this paper can accurately analyze the crack operation of track circuit, and

provide a novel method to realize transient analysis of track circuit.

\section{Acknowledgement}

This research was supported by the national Science foundation (No:51247002), the People's Republic of China and The Young Scholars Science Foundation of Lanzhou Jiao tong University (No:2015039)

\section{References}

1. Y.P. Zhang, B. Zhao, M.X. Tian: Transmittal Signal frequency of centre-fed ballastless track circuit. Journal of Southwest Jiao Tong University. 47(2012), No.7,7-11.
2. Y.P. Zhang, B. Zhao, M.X. Tian: Distance of centrefed boundless track circuits. Journal of the China Railway Society. 34(2012),No.9,65-69.

3. Q.M. Du, Y.P. Zhang, M.X. Tian, Y.Z. Min, Y. Ren: Real time detection method for broken rail . Journal of Lanzhou Jiao Tong University. 28(2009),No.9,123-126.

4. T. Sun, X.Z. Liu, Z.J. Jiang: An analytical solution of distortionless transmission line equations. Journal of Circuits and Systems. 12(2007), No.6,70-74.

5. Y.P. Zhang, B. Zhao, L. Wei: Analysis on the time responses of track circuits. Journal of the China Railway Society. 36(2014),No.9,68-72.

6. A. Orlandi, C.R. Paul; FDTD analysis of lossy multi-conductor transmission lines terminated in arbitrary loads.IEEE Trans.Electromagn.Compat. 38(1996), No.3,388-398.

7. J.A.Roden, C.R.Paul, W.T.Smith, S.D.Gedney: Finite difference time domain analysis of lossy transmissionlines.IEEETrans.Electromagn.Compat. 38(1996),No.1, 15-24.

8. T.D.Zhou,S.L.Dvorak,J.L. Prince: Lossy Transmission Line Simulation Based on closedFormtriangle impulse responses. IEEE Trans. Comput.-Aided Design Integr Circuits syst. 22 (2003),No.6,748-755.

9. E.C. Chang, S.M. Kang: Computationally efficient simulation of a lossy transmission line with skin effect by using numerical inversion of Laplace 
transform. IEEE Trans On CAS-I. 39(1992),No.11,861-868.

10. L.BRANCIK:Numerical Inversion of twodimensional Laplace transforms based on partial inversions. IEEE Trans. Electromagn.Compat.49(2007),No.1, 3-11.

11. Y.Y. Cao, H.J. Wu, Y.Q. Li: General solution of order in constant coefficient linear differential equations. Journal of Mathematics for Technology. 12(1996),No.2,156-158.

12. J. Jiang, C. H. Feng: A particular solution to the fourth order linear constant coefficient nonhomogeneous differential equations. Journal of Hechi university. 12 (1996),No.6,156-158.

13. S.P.Tian, H.L.Chen: Solving response of dynamic circuit in whole time domain. Journal of EEE. 31(2009),No.1,19-21

14. Z.S. Liu, L.Y. Zhu: Study on the response for higher-order linear circuits. Journal of Yangzhou University.1998, 1 (2):21-24. 1(1998),No.2,21-24.

15. B. Zhao, Y.P. Zhang: Modeling and Simulation of track circuit based on EMTP. Journal of Lanzhou University. 34(2015),No.3,81-86. 